\title{
OA2 (Free Re-Use)
}

Stevan Harnad

\section{Source}

Stevan Harnad. OA Primer for the Perplexed: I. (2008). Open Access

Archivangelism. http://openaccess.eprints.org/index.php?/archives/408-OA-Primer-for-

the-Perplexed-I.htmI

Articles or data made accessible/useable free online with various kinds of re-use licenses. 